\title{
Embolization of Superior Mesenteric Artery Aneurysm with Celiac Trunk Artery Occlusion - A Case Report
}

\section{Abstract}

Superior Mesenteric Artery Aneurym (SMAA) are a rare clinical finding with nonexistent or non-specific symptoms in most cases. The final event of SMAA is the rupture and its incidence is questionable. We present a case of a 61 year-old female with an accidental finding of giant SMAA.

Keywords: Superior mesenteric artery aneurym; Atherosclerosis; Infectious aneurysm; Computed tomography

Received: Septembre 06, 2017; Accepted: Septembre 21, 2017; Published: Septembre 28, 2017

\section{Introduction}

Visceral artery aneurysms (VAAs) are a rare entity in clinical practice, with a reported incidence ranging from $0.1 \%$ to $2 \%[1,2]$. SMAA is the fourth most frequent of the visceral aneurysms, corresponding 3 to $5 \%$ of the cases [3]. The infectious aneurysm is the most common etiology, however atherosclerosis has been growing as an importante cause. They are clinically important because of the high risk of rupture and death (up to $40 \%$ ), but the increased use of computed tomography (CT) and other imaging exams, the incidence of detected SMAAs is increasing, and more SMAAs are being diagnosed as asymptomatic $[3,4]$ We report a case of a pacient with accidental finding of SMAA and subsequently treated with embolization.

\section{Case Report}

A 61 year-old female, with a accidental finding of SMAA after a CT scan for investigation of renal calculus. The patient had no clinical symptoms and no findings at the physical examination related to the aneurysm. At the first time, we proceed with a angiotomography (Figures $\mathbf{1}$ and $\mathbf{2}$ ) for proper analysis, and the report was a $3.6 \times 3.0 \times 2.9$ saccular aneurysm with a suboclusion of the celiac trunk artery (CTA) with filling by the pancreatoduodenal artery (PDA) originated from the aneurysm. Due to the difficulty to confirm the image by the angiotomography slices, we decided to perform a diagnostic angiography.

With angiogaphy was found that the origin of the PDA was juxta neck of the aneurysm and a second arterial branch filling the CTA after the aneurysm (Figure 3). Because of the risk of hypoflow to

\section{Pietro de Almeida Sandri*, Joao Luiz Sandri, Felipe Machado dos Santos and Vinicius Lopes Adami}

\author{
CIAS, Hospital Unimed Vitória, Vitória/ES, \\ Brazil
}

*Corresponding author:

Pietro de Almeida Sandri

$\equiv$ pietrosandri@hotmail.com

CIAS, Hospital Unimed Vitória, Vitória/ES, Brazil.

Tel: 552733355000

Citation: Sandri PA, Sandri JL, Santos FM, Adami VL (2017) Embolization of Superior Mesenteric Artery Aneurysm with Celiac Trunk Artery Occlusion - A Case Report. J Vasc Endovasc Surg. Vol. 2 No. S1:26

the CTA, we decided to proceed with a embolization rather than use a covered stent. The total volume estimated of the aneurysm was 18.8 mililiters and it was used 8.39 meters of coil lenght, with a total of 15 coils (COSMOS VFC, Microvention Terumo, USA). The final result shows a patency of the PDA and CTA with no aneurysm filling (Figure 4).

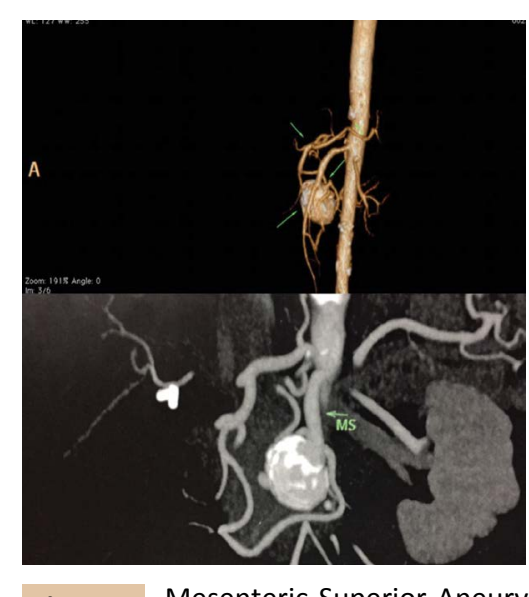

Figure 1 Mesenteric Superior Aneurysm with the CTA filling by the PDA. 


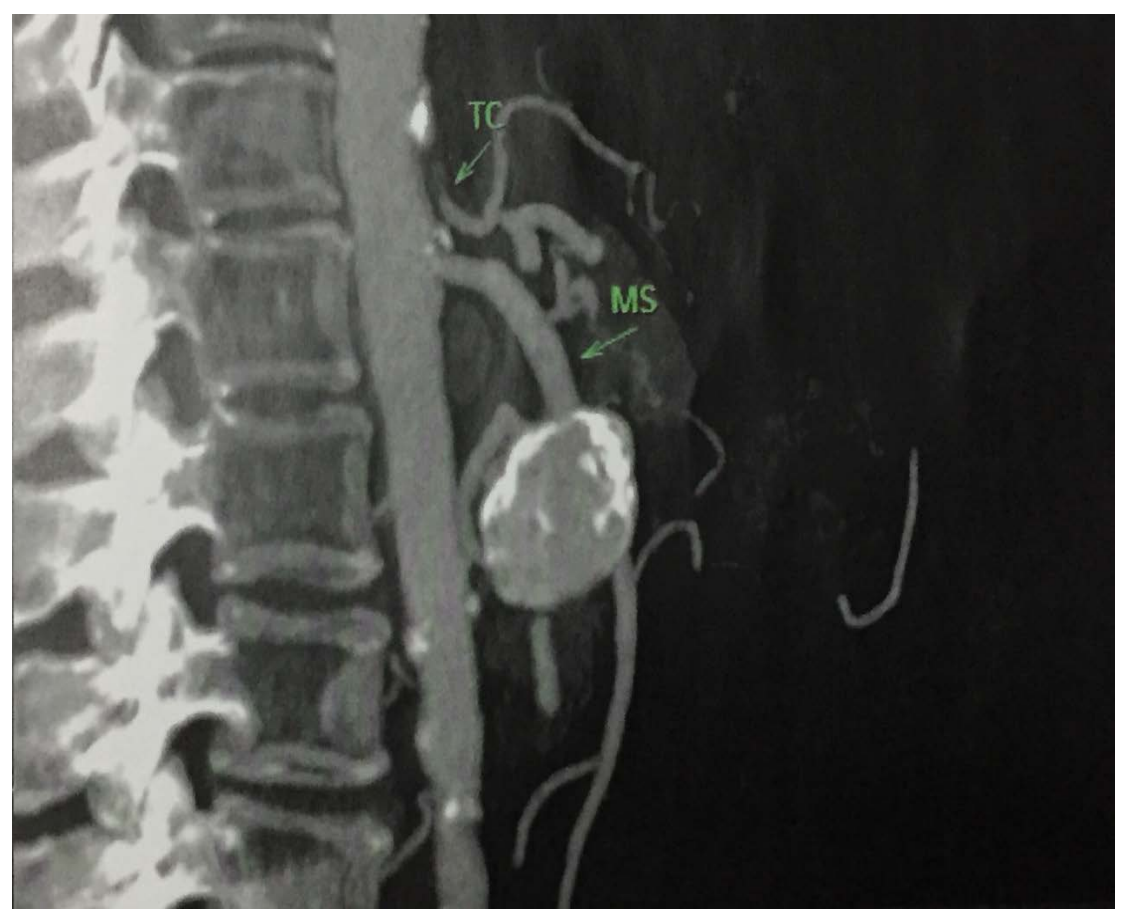

Figure 2 Superior Mesenteric Artery Aneurysm and the branchs of the superior mesenteric artery.

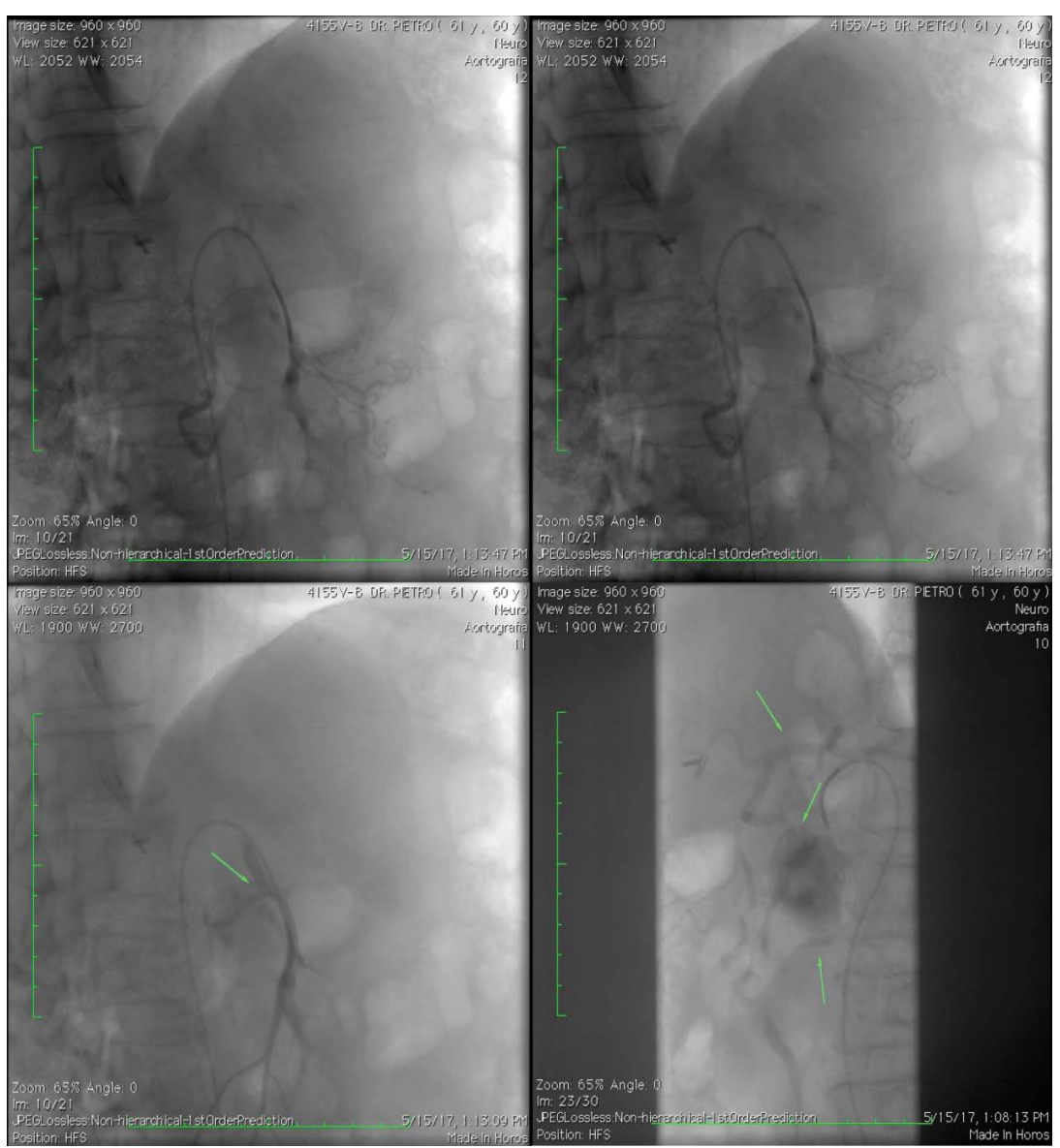

Figure 3 Angiography sequency showing the aneurysms and the arterial branchs from the superior mesenteric artery confirming the PDA origin from outside de aneurysm. 


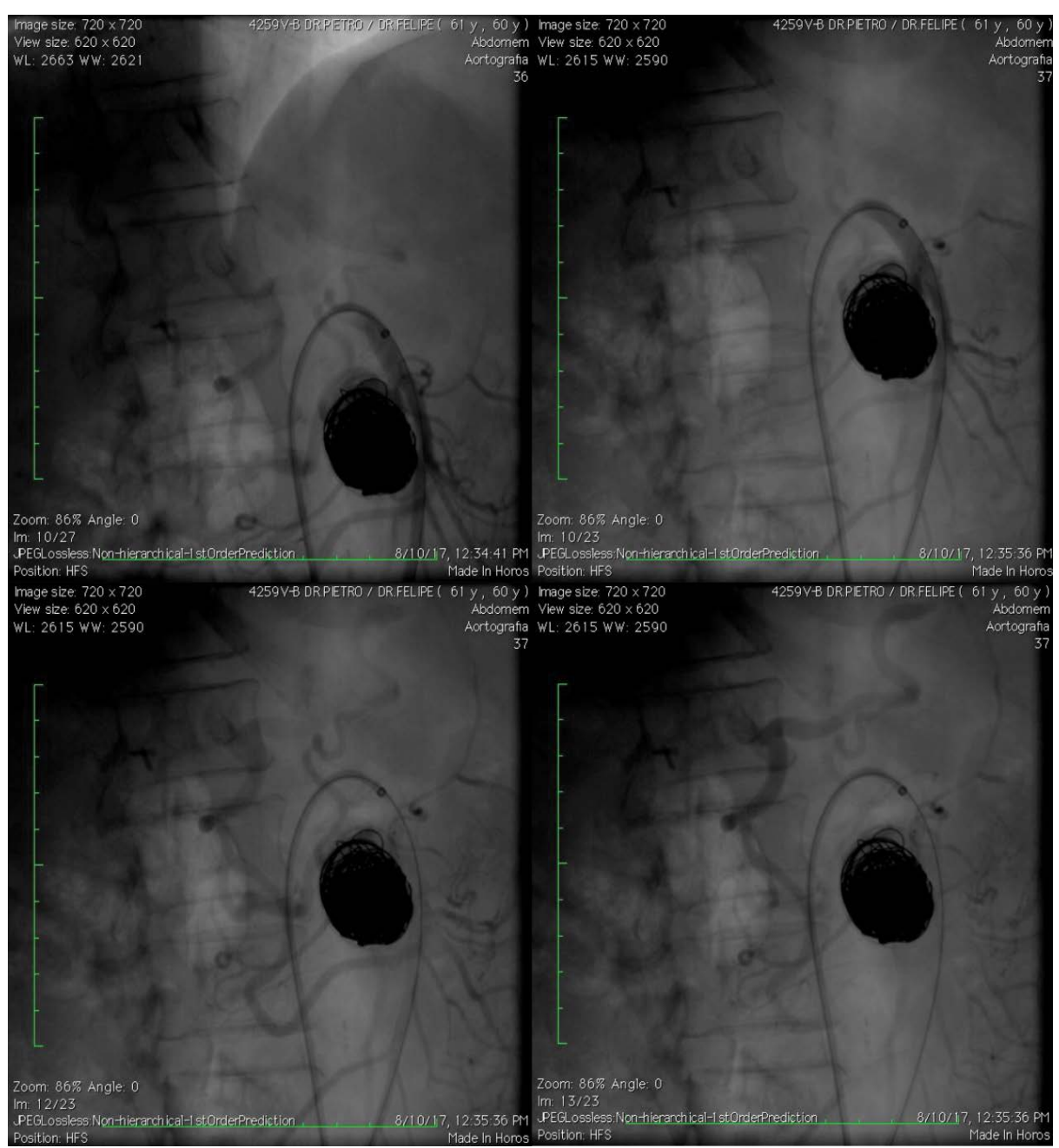

Figure 4 Final result after embolization, showing the patency of the superior mesenteric artery and the celiac trunk artery.

\section{Discussion}

Aneurysms of SMA are a rare condition [5]. This case describes a SMA aneurysm associate with a CTA sub oclusion and a branch from SMA filling the CTA. Historically, mycotic aneurysm have represented the majority of SMAA, but currently, the most frequent cause of aneurysms of the SMA is atherosclerosis [2]. Decades ago, $90 \%$ of all patients were symptomatic [6]; however, more recently, diagnosed SMAAs have been asymptomatic and discovered as incidental findings on abdominal imaging [2]. SMAA must be treated surgically or through endovascular approach, in the absence of complicating factors, due to the high risk of spontaneous rupture and thrombosis [7].

Open surgical interventions have been the main therapeutic option for VAAs. Given the necessity for exposure, open repair is associated with greater morbidity and mortality when compared to endovascular procedure [8].
Endovascular treatment can be performed using a covered stent or coil embolization with or without balloon or stent protection of main artery. In our case we decided to use a coil embolization due the arterial brach to the celiac artery and the format of the aneurysm. Corey et al. in a series of 264 splanchnic artery aneuryms, had 17 SMAA reported, being four with open repair and two repaired with endovascular therapy, and their recommendation is that all patients with a symptomatic or suspected mycotic SMAA $<25 \mathrm{~mm}$ can safely undergo surveillance. Asymptomatic SMAA $>25 \mathrm{~mm}$ should be considered for repair [8].

\section{Conclusion}

Currently, endovascular therapy with new materials, such as the nitinol detachable coils, balloons and stents for main artery protection are a valid option to open repair, but still requires further studies to determine long term durability of these procedures. 


\section{References}

1 Chadha M, Ahuja C (2009) Visceral Artery Aneurysm: Diagnosis and Percutaneous Management. Sem Interven Radiol 26: 196-206.

2 Hoogendorne W, Schlosser FJF, Sumpio BE (2013) A Giant Superior Mesenteric Artery Aneurysm Mimicking a Abdominal Aortic Aneurysm. Aorta 1: 52-56.

3 Jiang J (2011) Therapeutic Management of Superior Mesenteric Artery Aneurysms. J Vasc Surg 53: 1619-1624.

4 Lindemberg CG, Stribeck H (1992) Aneurysms of Superior Mensenteric Artery and it Branches. Gastrointestinal Radiology 17: 132-134.
5 Dasari BV, Mullan M, Lau L, Loan W, Lee B (2013) A 6.5-cm Pseudoaneurysm of the Superior Mesenteric Artery Managed by Primary Surgical Repair. Vascular 21: 39-42.

6 Venyo AK, Kodzo A, Venyo G (2011) Enlarging Superior Mesenteric Artery Aneurysm Successfully Treated By Selective Angiography and Embolization With Terumo Hydrocoil: A Case Report And Review Of The Literature. WebMedCentral Radiology.

7 Bowens NM, Wang GJ (2014) Endovascular Repair of an SMA Aneurysm With Complex Aortic Pathology. Endovascular Today 62-64.

8 Corey MR, Ergul EA, Cambria RP, English SJ, Patel VI, et al. (2016) The natural history of splanchnic artery aneurysms and outcomes after operative intervention. J Vasc Surg 63: 949-957. 